\title{
El transporte marítimo y los desarrollos portuarios. El nuevo rol de las alianzas marítimas en el tablero internacional
}

\author{
FERNANDO GONZÁLEZ LAXE \\ Universidad de A Coruña, Facultad de Economía y Empresa, Campus de Elviña, s/n, 15071 A Coruña, \\ España.E-mail: laxe@udc.es.
}

\begin{abstract}
RESUMEN
El transporte intermodal ha permitido a los sistemas de transporte marítimo compensar las dificultades de conexión entre varios puntos. Emergen cadenas de transporte que se basan principalmente en el interface marítimo/ferroviario, permitiendo ampliar la capacidad de distribución y ofertando itinerarios regulares y con tarifas predeterminadas. Los puertos se convierten en espacios de convergencia entre los sistemas de transportes, en suministradores de servicios y se integran en un sistema de distribución de mercancías que requieren de desarrollos logísticos. Nuestra aportación reseña las distintas formas de alianzas que han adoptado las compañías navieras que operan en el transporte marítimo regular; los acuerdos de cooperación y las alianzas entre las mismas; y determinamos los distintos niveles de jerarquía de los puertos atendiendo a la conformación y al desarrollo de las compañías de transporte marítimo en lo concerniente a las rutas y a los modelos portuarios.
\end{abstract}

Palabras Clave: Transporte marítimo; logística marítima; modelos portuarios; alianzas empresariales.

\section{The Maritime Transport and the Port Development. The New Role of the Maritime Alliances in the International Board}

\begin{abstract}
The intermodal transport has allowed to the system of maritime transport to compensate the difficulties of connection between several points. They emerge the chains of transport based, principally, on the maritime-railway interface, which extend the distribution capacity covering new itineraries with predetermined rates. The ports turn into spaces of convergence between the systems of transport; offer services and join distribution systems that they need of logistics developments. The works explains the different forms of alliances and agreements of collaboration that sign the shipping companies. It calls the attention on the different levels of port hierarchy attending to the development of the maritime routes and to the port models.
\end{abstract}

Keywords: Maritime Transport, Port Logistics, Maritime Alliances.

Clasificación JEL: F02, L91, R41. 


\section{INTRODUCCIÓN: LOS CAMBIOS EN LOS MODELOS PORTUARIOS}

En los últimos años, y más concretamente a partir de los años 90, hemos asistido a cambios muy profundos en lo que concierne a los patrones de organización de las actividades portuarias. La UNCTAD (1992) al delimitar los modelos conceptuales de los puertos establecía tres criterios clave: políticas de desarrollo portuario en lo que atañe a las estrategias y actividades; alcance y extensión de las actividades portuarias; e integración de las actividades. Dicha clasificación nos aportaba las posibilidades de establecer una secuencia de tres generaciones.

La primera generación, previa a los años sesenta, se caracterizaba por el hecho de que los puertos operaban de manera aislada y actuaban como interface entre el espacio terrestre y el transporte marítimo. De esta manera, el puerto permanecía desligado de las actividades comerciales y de transporte; esto es, presenciábamos un aislamiento entre el puerto y su área circundante, sin apenas cooperación entre las mismas, Finalmente, las distintas compañías que operaban en el puerto lo hacían de manera independiente, no llevando a cabo operaciones conjuntas a la hora de promocionar el puerto a nivel comercial.

Los puertos de segunda generación contemplaban una amplia gama de funciones y actuaban como centros de servicios comerciales, industriales y de transporte. El alcance de la actividad del puerto se extiende hasta las actividades comerciales, "añadiendo valor" a las cargas. Al contrario de los puertos de primera generación, los de segunda muestran una estrecha relación entre los socios comerciales y del transporte con las áreas próximas al puerto. O sea, los puertos no actúan de manera aislada, sino en relación con la industria del transporte. (Hoyle \& Hilling,1984).

Los puertos de tercera generación, década de los noventa, se inscriben en la era de la globalización. Se convierten en nodos dinámicos dentro de la compleja red internacional de producción/distribución. La gestión portuaria se caracteriza por el desarrollo de centros integrados de transporte y por la creación de plataformas logísticas. Los servicios portuarios se especializan, se hacen más variables y combinan multitud de servicios y prestaciones. Se adaptan rápidamente a los avances tecnológicos y de los equipamientos. Se crean áreas industriales para generar mayores rendimientos de las cargas con respecto a los puertos y se refuerzan las medidas de protección medioambiental y de seguridad. Finalmente, en los puertos de tercera generación se produce una notable mejora en lo que atañe a la eficiencia administrativa al mejorar y uniformizar los documentos administrativos y burocráticos. 
Tabla 1

Tipologías de los modelos portuarios de tres generaciones

\begin{tabular}{|l|l|l|l|}
\hline \multirow{2}{*}{$\begin{array}{l}\text { Estrategia } \\
\text { desarrollo portuario }\end{array}$} & $\begin{array}{c}\text { PRIMERA } \\
\text { GENERACIÓN }\end{array}$ & $\begin{array}{c}\text { SEGUNDA } \\
\text { GENERACIÓN }\end{array}$ & \multicolumn{1}{c|}{$\begin{array}{c}\text { TERCERA } \\
\text { GENERACIÓN }\end{array}$} \\
\hline $\begin{array}{l}\text { Carga granel; punto de } \\
\text { intercambio transporte. }\end{array}$ & $\begin{array}{l}\text { Graneles secos y líquidos; } \\
\text { Transporte, centro } \\
\text { comercial e industrial. }\end{array}$ & $\begin{array}{l}\text { Carga granel y contenerizada; } \\
\text { Centro transporte } \\
\text { integrado/plataforma logística } \\
\text { para el comercio } \\
\text { internacional. }\end{array}$ \\
\hline $\begin{array}{l}\text { Alcance } \\
\text { actividades }\end{array}$ & $\begin{array}{l}\text { Carga, descarga, } \\
\text { almacenaje, servicios } \\
\text { navegación; muelles y } \\
\text { áreas marítimas } \\
\text { limítrofes. }\end{array}$ & $\begin{array}{l}\text { Carga, descarga, } \\
\text { almacenaje, servicios } \\
\text { navegación; } \\
\text { transformación carga: } \\
\text { servicios industriales y } \\
\text { comerciales relacionados } \\
\text { con el buque; mayor área } \\
\text { portuaria. }\end{array}$ & $\begin{array}{l}\text { Carga, descarga, almacenaje, } \\
\text { servicios navegación; } \\
\text { transformación de la carga; } \\
\text { distribución de la carga y la } \\
\text { información; actividades } \\
\text { logísticas; Terminales y } \\
\text { extensión del puerto hacia } \\
\text { tierra. }\end{array}$ \\
\hline $\begin{array}{l}\text { Características } \\
\text { organizativas }\end{array}$ & $\begin{array}{l}\text { Actividades } \\
\text { independientes dentro del } \\
\text { puerto; relación informal } \\
\text { entre el puerto y sus } \\
\text { usuarios. }\end{array}$ & $\begin{array}{l}\text { Mayor relación puerto y } \\
\text { usuarios; escasa relación } \\
\text { entre actividades } \\
\text { portuarias; poca relación } \\
\text { entre puerto y municipios } \\
\text { limítrofes. }\end{array}$ & $\begin{array}{l}\text { Unidad de la comunidad } \\
\text { portuaria; integración puerto } \\
\text { por medio de cadena de } \\
\text { comercio y transporte; } \\
\text { estrecha relación entre puerto } \\
\text { y áreas; mayor organización } \\
\text { portuaria. }\end{array}$ \\
\hline $\begin{array}{l}\text { Características de } \\
\text { producción }\end{array}$ & $\begin{array}{l}\text { Flujo de carga; servicio } \\
\text { individual; bajo valor } \\
\text { añadido. }\end{array}$ & $\begin{array}{l}\text { Flujo de carga; } \\
\text { transformación de la } \\
\text { carga; servicios } \\
\text { combinados; mejora del } \\
\text { valor añadido. }\end{array}$ & $\begin{array}{l}\text { Flujo información y carga; } \\
\text { package de servicios múltiple; } \\
\text { alto valor añadido; tecnología } \\
\text { /Know-how. }\end{array}$ \\
\hline
\end{tabular}

Fuente: Elaboración propia a partir UNCTAD, 1992.

Las economías portuarias actúan bajo varios vectores agrupándose, esencialmente, en tres estrategias. Una estrategia de concentración, en lo que se refiere a la capacidad de atracción y establecimiento de las sedes de armadores y agentes, con el objetivo de lograr una progresiva reducción de costes y una creciente concentración de tráficos. Una estrategia de intensificación del transporte, que afecta al número, tipo y tamaño de las embarcaciones, especialmente porta-contenedores, así como el establecimiento de acciones que reduzcan los tiempos de estancia, incrementen las calidades de los servicios ofertados y que amplíen los trayectos directos con otros puertos y áreas geográficas. Y, finalmente, una estrategia de conjunto e integración que, basada en la intermodalidad, sea capaz de desarrollar los equipamientos, instalaciones, plataformas logísticas y redes necesarias para el establecimiento de prioridades en lo que respecta a la calidad de los servicios, valorizando su posición geográfica y los ejes del comercio internacional.

Se apuntan dos corrientes de análisis para enfocar dichas estrategias. La primera, desde la perspectiva de la mercancía, en donde el papel de los puertos consiste en redistribuir la carga; integrar y combinar los distintos modos de transporte terrestre y marítimo; mejorar e impulsar la calidad del intercambio y de la distribución; y responder a las nuevas funciones de estocage y de instalaciones 
portuarias especializadas, tales como los equipamientos portuarios, la proliferación de áreas diferenciadas y la existencia de instalaciones apropiadas para cargas líquidas, mercancías sólidas y combinadas, junto a medios tales como grúas, pórticos, contenedores, automatización de terminales, etc. que constituyen la esencia de la consolidación de un hub. El segundo análisis, se lleva a cabo desde la relación con el buque y, entonces, las funciones portuarias requieren de la existencia de medios imprescindibles para garantizar la presencia de las unidades de carga, tales como torres de control, acceso, circulación y guía; remolcadores, capacidad de maniobra, capacidad para la inmovilización del barco, suministros, energía, agua, reparación naval, son buenos ejemplos de los nuevos e imprescindibles requisitos.

La "revolución logística" en los transportes marítimos y en las estructuras organizativas portuarias han obligado a los puertos a equiparse en terminales especializadas y con nuevas instalaciones. En la medida que el transporte se realiza sin ruptura de carga, por uno o varios medios de transporte; dado que es más fácil de manipular, de llenar, de vaciar y de almacenar; y porque obtiene una mayor productividad, en la medida que un muelle de contenedores puede albergar y movilizar 10 veces más cargas/mercancías que un muelle clásico, las inversiones en infraestructuras portuarias se prevén más exitosas que en otras actividades.

Muchas estructuras portuarias se convierten en plataformas de intercambio de mercancías; y en su desarrollo se formalizan los hub's marítimos (nudos de redes) que albergan a los navíos-madre (mothers ships) que alimentan a los portacontendores de talla media (feeders), conformando un nuevo equilibrio: polo/núcleo central + plataforma logística. De ahí, los cambios en la valorización de las instalaciones portuarias (Ducruet \& Zaidi, 2012),

Los puertos se reafirman, por un lado, como "nodos funcionales" que ejercitan funciones de atracción y de captación de tráficos; y, por otro, como impulsores de una dinámica de desarrollo territorial específica para seducir y desviar flujos de mercancías (Guerrero \& Rodrigue, 2014).

Los nuevos objetivos portuarios se centran, en consecuencia, en: a) atraer nuevos tráficos de mercancías; b) interconectar áreas económicas y formalizar corredores de transporte; c) captar inversiones para el impulso económico y territorial; d) estimular las interfaces "tierra-mar"; y e) en adaptarse, de manera permanente, a las nuevas normas institucionales y de regulación económica. La nueva dinámica se completa, asimismo, con una especialización de los desarrollos portuarios y con un proceso de mayor partenariado y de privatizaciones que acentúa la rivalidad y competencia. 


\section{LOS TRÁFICOS MARÍTIMOS Y ACTUACIÓN DE LOS OPERADORES}

Este modelo cambiante en lo que se refiere a los niveles de tráficos marítimos y en la actuación de los operadores permite destacar cuatro elementos relevantes. En primer lugar, las compañías navieras están cada vez más involucradas en las operaciones de las terminales. En segundo término, los operadores internacionales desean adquirir concesiones para albergar nuevas terminales. En tercer aspecto implica un nuevo proceso de oferta de normativas sobre los arrendamientos y las concesiones, emergiendo un nuevo mercado de concesiones de terminales. Y, finalmente, surgen nuevas formas de financiación y de situaciones de partenariado entre lo gubernamental y lo privado.

Los puertos, como nodos de transferencia de flujos, se constituyen a la vez como centros de distribución e integración de cargas y contribuyen a revalorizar la localización geográfica en la que se adscriben, al potenciar las zonas de influencia territoriales. Los nuevos conceptos y las funciones tradicionales de los puertos hacen que se conviertan en "pasillos" o en "pivotes", al objeto de eliminar el concepto de almacenaje y proporcionar nuevos servicios que contribuyen a "añadir valor a las mercancías", pues se ha considerado al puerto como "una parte de la cadena logística”. (Heaver, 2002; Haralambides, 2017; Hirata, 2017).

En el actual milenio, y más en concreto después de la crisis de 2008, se abren nuevas expectativas en el ámbito de las operaciones y sistemas portuarios. Varios son los ejes que alteran los comportamientos anteriores.

- Los tipos de carga. Las distintas modalidades de carga se modificaron a lo largo de las últimas décadas. La creciente especialización y automatización ha sido claves en las operaciones de carga/descarga de los puertos. La unitización de las mercancías sobre la bases de los contenedores ha revolucionado los transportes y exigen fuertes demandas de inversión tanto en el tamaño de los buques (que han aumentado) como en las infraestructuras de los puertos (que reclaman mayores dragados y sistemas de tecnologías de información que apoyen los movimientos de los contenedores). Estas nuevas condiciones de proceso del manejo de mercancías suponen la necesidad de construir nuevas terminales, que son preciso situarlas fuera ó en el exterior de los recintos portuarios tradicionales; y, también se pone de manifiesto la necesidad de desarrollar sistemas secundarios e integrados que conviertan al puerto en plataformas logísticas, y no solo en puntos de tránsito de las mercancías (Notteboom \& Rodrigue, 2012),

Hesse \& Rodrigue (2004) reflejan la nueva dimensión geográfica de la logística portuaria y establecen las distintas cadenas de valor integradas a partir de los transportes marítimos y las operaciones de carga/descarga de 
mercancías en los puertos, llamando la atención sobre los conceptos de los nodos/redes y los conceptos de fricción en la logística del transporte.

- La propiedad de los puertos. Hasta el principio de la década de los ochenta la propiedad de las infraestructuras portuarias ha correspondido al sector público, a excepción del Reino Unido donde existían varios puertos privados. A partir de los años 80, del pasado siglo, comienza a descomponerse la propiedad de la superestructura y las operaciones portuarias (Brooks et al., 2017). Al aumentar el interés de sector privado en las operaciones portuarias asistimos a nuevas dinámicas de combinación de métodos alternativos en lo que concierne a la gestión, incrementándose, en primer lugar, la participación del sector privado y, en segundo término, una mayor asignación de tareas y funciones a empresas relacionadas con las actividades marítimas y/o de transporte. Prueba de ello son los mayores niveles de participación privada y su presencia es cada vez mayor en la propiedad de las terminales portuarias (Brooks, 2004). Dicho proceso presenta, asimismo, una triple característica: a) cada vez es más rápido e intenso; b) han surgido actores muy importantes y relevantes procedentes del grupo de las compañías internacionales; y c) el accionariado es cada vez más complejo e internacional. De ahí que dichos cambios puedan alterar los procesos de inversión y de financiación, a la vez que emergen dinámicas de construir/operar/transferir (build-operate-transfer) las terminales portuarias.

- Cambios notables en las funciones portuarias y en su aportación al desarrollo económico. Hasta hace poco tiempo la función portuaria no estaba considerada como esencial en los procesos de desarrollo. En la actualidad, en la medida que aceptamos la integración de los puertos en cadenas logísticas globales y que los puertos operan bajo el contexto de movimientos de carga que poseen efectos sinérgicos y externalidades positivas en áreas próximas al recinto portuario, resulta obvio la relevancia que adquieren el área portuaria y su vinculación con el hinterland asociado (Ng, 2018). O sea, se pasa de un transporte segmentado a uno integrado. Las mercancías se transportan de manera unitizada. Las compañías actúan con una mayor integración (vertical y horizontal). Existe una tendencia hacia la concentración de mercancías en pocos puertos y las funciones portuarias se hacen más complejas, se amplían las relaciones entre puertos y usuarios, creándose las Maritime Industrial Development Areas (MIDAS).

Estas circunstancias impulsaron a los puertos a expandirse fuera de sus tradicionales recintos. Requieren más extensión, tienden a especializarse, deben ofertar más servicios, se obligan a integrarse en cadenas de transporte dependiendo de las cargas y de las necesidades de los clientes, y se asiste a una mayor rivalidad y competitividad portuaria. 
Entre los rasgos más sobresalientes de la globalización marítima destacamos, en consecuencia, los siguientes: a) la utilización del contenedor, como instrumento de transporte, ha supuesto una auténtica revolución y su crecimiento ha sido espectacular; b) el tamaño de los buques ha aumentado en la medida que se demandan embarcaciones capaces de transportar más carga; c) cada vez son mayores las mercancías que se transportan; y d) cada vez son más numerosos los puertos integrados en la distribución de mercancías y en la inserción en los tráficos marítimos a nivel internacional.

Después de unos años de estancamiento en los que refiere a los movimientos de contenedores como consecuencia de los efectos producidos por la crisis del 2008, los tres últimos años han registrados tasas de crecimiento positivas: 1,4\%, en 2015; 2,5\%, en 2016; y 6,5\%, en 2017. El volumen manejado en los 110 puertos de contenedores más relevantes del mundo aumentó un 6,1\% en 2017, merced a la mejoría de la economía mundial y a la reactivación del comercio internacional. Los mencionados 110 puertos más relevantes manejaron alrededor de 600 millones de Teus en 2017, frente a los 565 millones de Teus, contabilizados en 2016. De dichos 110 puertos, 98 registraron mejorías y solo 12, mostraron descensos,

El análisis de los movimientos de contenedores por zonas del mundo pone de manifiesto la concentración de los tráficos marítimos, en donde el continente asiático es el núcleo principal. Desagregando los tráficos de contenedores atendiendo a las rutas cabe señalar que los itinerarios con origen en el continente asiático son los que registran mayores volúmenes. Esto es, las rutas transpacíficas y las que vinculan Asia/Europa son las que dominan los tráficos mundiales; y más en especial en las direcciones Este/Oeste, Por el contrario, la ruta transatlántica, la que une el continente europeo y el americano, va perdiendo importancia a lo largo de este último decenio en relación a las otras dos rutas anteriormente mencionadas.

Estas dinámicas permiten visualizar con mayor nitidez los procesos de concentración y polarización geográfica, así como los centros de actividad económica vinculados con los movimientos de mercancías.

Tabla 2

Evolución del tráfico de contenedores atendiendo a las principales rutas marítimas (expresado en millones de Teus)

\begin{tabular}{|l|c|c|c|c|c|c|c|c|}
\hline & $\mathbf{1 9 9 5}$ & $\mathbf{2 0 0 0}$ & $\mathbf{2 0 1 0}$ & $\mathbf{2 0 1 1}$ & $\mathbf{2 0 1 2}$ & $\mathbf{2 0 1 3}$ & $\mathbf{2 0 1 4}$ & $\mathbf{2 0 1 5}$ \\
\hline Transpacífica & 8 & 11 & 19 & 19 & 20 & 22 & 23 & 24 \\
\hline Asia/ Europa & 4 & 7 & 19 & 20 & 20 & 22 & 22 & 22 \\
\hline Transatlántica & 2 & 4 & 6 & 6 & 6 & 6 & 7 & 7 \\
\hline
\end{tabular}

Fuente: UNCTAD. Revista de Transporte Marítima. 
Una de características más sobresalientes de los últimos años es el incremento del tamaño de los buques (Merk et al., 2015). Dicho fenómeno, denominado "gigantismo naval", significa que cada año los buques en funcionamiento son capaces de albergar mayores volúmenes de mercancías y generar mayores economías de escala. Dicho proceso se ha acelerado en el último quinquenio y todavía la ingeniería está desarrollando nuevos prototipos de mayor envergadura y desarrollo técnico, sin que existe tope por el momento se nos atenemos a las informaciones proporcionadas por los astilleros.

Tabla 3

Evolución tamaño de buques

\begin{tabular}{|c|c|c|c|}
\hline Nombre buque & Naviera & Capacidad teus & Fecha operativa \\
\hline Marco Polo & CMA/CGM & 16.000 & 2012 mayo \\
\hline Mc-Kinney Moller & Maersk & 18.270 & 2013 abril \\
\hline Globe & COSCO & 18.992 & 2013 Junio \\
\hline Oscar & MSC & 19.224 & 2014 Noviembre \\
\hline Barzan & UASC & 18.830 & 2015 abril \\
\hline Triumph & MOL & 20.170 & 2017 marzo \\
\hline Madrid & Maersk & 20.568 & 2017 abril \\
\hline Hong Kong & OOCL & 21.413 & 2018 mayo febrero \\
\hline A. Saint Exupery & CMA/CGM & 20.600 & 2018 febrero \\
\hline Truth & MOL & 20.182 & \\
\hline
\end{tabular}

Fuente: Elaboración propia a partir de las noticias suministradas por las empresas.

Slack, Comtois y McCalla (2002) especifican que el proceso de globalización ha supuesto una extensión y una nueva re-elaboración de las redes de comunicación marítima merced a la emergencia de nuevos mercados y a la intensificación de las relaciones mercantiles entre las economías desarrolladas y las de los países en desarrollo. A tal punto se desarrolló esta tendencia que las primeras alianzas de líneas regulares han incrementado tanto los servicios como la cooperación en los servicios conjuntos entre las empresas. Por ejemplo, en 1989 el número de servicios regulares que prestaban los grupos individuales como Maersk-Sealand; CCMA/CGM y Evergreen; junto a las pioneras Alianzas como las denominadas, en aquel entonces, Gran Alliance (compuesta por Hapag; P\&O Nedlloyd; MISC; OOCL; y NYK); la United Alliance (integrada por Hanjin; DSR Senator; y Cho-Yang); la New World Aliance (de la que eran miembros la Hyundai Merchant Marine; APL; NOL; y MOL) junto a las empresas K-Line, COSCO y Yan Ming, sumaban un total de 422 servicios; y diez años más tarde, en 1999 ascendía a 545. En la actualidad, resulta muy difícil estimar el conjunto de servicios ofertados por las compañías navieras dadas las múltiples interrelaciones con otras actividades económicas, comerciales y de servicios. Es decir, dicha mayor oferta de servicios no solo se identifica 
asumiendo un mayor número de buques, un mayor tamaño de las embarcaciones y una mayor especialización de las unidades; sino que se amplía al campo de la logística, del transporte terrestre, los seguros, la industria naval, .

El proceso de concentración y de polarización geográfica se acentúa de manera progresiva en los últimos años. Las diez primeras compañías navieras operadoras alcanzan los 3.125 buques; o sea, el 51\% del total mundial y alrededor del $81 \%$ de la capacidad del transporte. Sus cifras son más elevadas que las contabilizadas el año anterior ( $71 \%$ de la cuota de mercado); o hace seis años, cuando su cuota de mercado apenas llegaba al $60 \%$ de la misma. Más relevante es que las tres primeras compañías navieras (Maersk; MSC; y CMA/CGM) suman, en el año 2017, el 45,2\% del total de la capacidad mundial, cinco puntos porcentuales por encima de lo obtenido en 2016.

Por lo que hace referencia a la dominación asiática, se comprueba que 6 de las 10 primeras compañías tienen su sede en Asia; las cuatro restantes son europeas; en tanto que las norteamericanas mantienen una presencia muy reducida.

Tabla 4

Cuota de mercado de las diez principales navieras

\begin{tabular}{|c|c|c|c|c|c|}
\hline Empresa & Sede & $\begin{array}{c}\text { Número } \\
\text { Barcos }\end{array}$ & $\begin{array}{c}\text { Capacidad } \\
\text { Teus }\end{array}$ & $\begin{array}{c}\text { Cuota mercado } \\
\text { mayo 2018 }\end{array}$ & $\begin{array}{c}\text { Cuota mercado } \\
\text { 2002 (**) }\end{array}$ \\
\hline Maersk & Dinamarca & 756 & 4.143 .281 & $18,8 \%$ & $15,8 \%$ \\
\hline MSC & Suiza & 519 & 3.258 .231 & $14,8 \%$ & $13,8 \%$ \\
\hline CGM/CMA & Francia & 493 & 2.524 .484 & $11,4 \%$ & $8,2 \%$ \\
\hline COSCO, & China & 358 & 1.962 .507 & $8,9 \%$ & $4,1 \%$ \\
\hline Hapag Lloyd & Alemania & 228 & 1.616 .674 & $7,3 \%$ & $3,9 \%$ \\
\hline ONE $\left(^{*}\right)$ & Japón & 232 & 1.550 .163 & $7,0 \%$ & $3,8 \%$ \\
\hline Evergreen & Taiwan & 197 & 1.089 .704 & $4,9 \%$ & $2,5 \%$ \\
\hline OOCL & China & 100 & 694.597 & $3,1 \%$ & $2,1 \%$ \\
\hline Yang Ming & Taiwan & 107 & 646.393 & $2,9 \%$ & $1,5 \%$ \\
\hline PIL & Malaysia & 135 & 427.624 & $1,9 \%$ & $58,2 \%$ \\
\hline Top 10 & & 3.125 & 17.910 .658 & $81,0 \%$ & \\
\hline
\end{tabular}

$\left(^{\star}\right)$ ONE (Ocean Network Express) es la entente empresarial llevada a cabo entre las navieras japonesas de NYK, K-Line y MOL, operativa a partir del 1 de abril de 2018.

$\left.{ }^{(*}\right)$ En 2012, en el top-10 estaban incluidos Hamburg Süd (adquirida por Maersk) y United Arab Sh. Co (UASC) adquirida por Hapag-Lloyd.

Fuente: Alphaliner.

Esta mayor intensificación de servicios y el mayor número de buques se traduce en dos vectores relevantes. El primero, supone afianzar la posición de liderazgo de las primeras empresas navieras de líneas regulares. Esto es, Maersk, MSC, CGM/CMA y COSCO, refuerzan sus primeras posiciones en el ranking mundial. En segundo lugar, se aprecia una mayor capacidad para conformar alianzas y para fortalecer los procesos de fusiones y adquisiciones de empresas, 
tal y como se pone de manifiesto tanto por las adquisiciones llevada a cabo (APL fue adquirida por CMA/CGM; Hamburg Süd fue adquirida por Maersk; CSG fue adquirida por COSCO; UASC fue adquirida por Hapag-Lloyd); como en las alianzas estratégicas constituidas (ONE es el producto de la entente entre las tres navieras japonesas: Nippon Yussen Kabushiki (NYK); Kawasaki Kisen Kaisha (K-Line); y Mitsui OSK Line (MOL).

La cooperación entre compañías se produce de varias formas:

a) por la constitución de alianzas y la formación de grupos marítimos;

b) por el desarrollo de marcos de cooperación entre compañías para la formalización de nuevas rutas regulares de transporte marítimo; y

c) por la constitución de filiales dentro de cada compañía. Este proceso de integración vertical es continuo y sostenido en la última década, dinámica que ha acentuado el proceso de concentración empresarial.

Tabla 5

Dinámica de constitución de Alianzas Marítimas

\begin{tabular}{|c|l|l|}
\hline Año & \multicolumn{1}{|c|}{ Alianza } & \multicolumn{1}{c|}{ Miembros de la Alianza Marítima } \\
\hline 1994 & Global Alliance & APL; MOL; OOCL; Nedlloyd. \\
\hline 1995 & Gran Alliance & Hapag-Lloyd; NYK; NOL; P\&O \\
\hline 1998 & New World Alliance & APL; MOL; Hyundai Merchant Marine \\
\hline 2000 & CKYH Alliance & COSCO; K-Line; Yang Ming; Huyndai \\
\hline 2011 & G'6 & APL; MOL; Hyundai; Hapag-Lloyd; NYK; OOCL. \\
\hline 2014 & 2 M & Maersk; MSC. \\
\hline 2014 & Ocean-3 Alliance & CSG; CMA/CGM; UASC. \\
\hline 2014 & CKYHE Alliance & COSCO; K-Line; Yang Ming; Hanjin; Evergreen. \\
\hline 2017 & Ocean-3 Alliance & CMA/CGM (+ APL); COSCO (+ CSG); Evergreen; OOCL. \\
\hline 2017 & $\begin{array}{l}\text { THE Alliance (Transport } \\
\text { High Efficiency = THE) }\end{array}$ & Yang Ming; Hapag-Lloyd (+ UASC); ONE (NYK + MOL+ K-Line). \\
\hline
\end{tabular}

Fuente: Elaboración propia.

Tabla 6

Composición actual de las Alianzas

\begin{tabular}{|l|l|}
\hline $2 \mathrm{M}$ & Maersk + MSC \\
\hline Ocean Three & CMA/CGM + COSCO + Evergreen + OOCL \\
\hline THE Alliance & Yang Ming + Hapag Lloyd + ONE \\
\hline
\end{tabular}

Fuente: Elaboración propia.

Del mismo modo, una segunda tendencia viene dada por la relevancia de las terminales marítimas, cuyo objetivo principal radica en servir de nexo para que las cargas/descargas sean rápidas (lograr tiempos inferiores a 24 horas, por ejemplo, se ha convertido en un fin para las terminales más eficientes) y que puedan fomentar su integración en las cadenas logísticas. 
¿Qué se busca en ambos casos? Principalmente: a) maximizar el tonelaje transportado y las mercancías movidas; b) maximizar el valor añadido de las mercancías movidas; y c) maximizar las oportunidades que generan los servicios en el hinterland.

\section{LA ARTICULACIÓN DE LAS EMPRESAS}

El seminal estudio de Frémont y Soppé (2004) ha servido para resaltar las distintas estrategias sostenidas por las empresas de líneas regulares marítimas. Sus diferentes procesos de diferenciación se fundamentan en la obtención de ventajas comparativas duraderas; en proporcionar estrategias identificativas diferenciadas; y en conformar redes marítimas a nivel global. Analizando los servicios marítimos, la utilización de los puertos, la capacidad total de las unidades y la frecuencia de los servicios, o sea las capacidades de transporte y los puertos que están vinculadas a dichas líneas, revelan que los esquemas finales muestran que la circulación de los flujos de mercancías subrayan un sistema global, fuertemente jerarquizado y estructurado por las estrategias de las empresas de líneas regulares; en el que Asia es el principal polo de dicho sistema (El Kalla et al., 2017; Ducruet, 2016); González-Laxe et al.,2012).

Las alianzas entre grandes empresas, previamente mencionadas, constituyen el rol determinante de dicha estructuración de las redes marítimas globales en la medida que seleccionan los puertos (cada vez más selectivos y específicos); concentran la actividad en las rutas Este-Oeste; y se adecuan permanente a las distintas estrategias de adaptación y de participación en la globalización (Yeo, 2013, 2015; Sys et al., 2011).

Varios modelos sirven de explicación del dinamismo de ciertos grupos empresariales y a las estrategias de desarrollo económico nacional de ciertos países. Dos consideraciones iniciales quedan claras: la dominación asiática y la renovación europea (Tourret, 2004). En lo que respecta a la primera, destacaremos las distintas opciones llevadas a cabo atendiendo a los distintos países. En primer término, Japón quiere hacer frente a la competencia china, por lo que se a partir del 1 de abril de 2018, entra en el mercado marítimo la nueva agrupación empresarial denominada ONE, producto de la conjunción de ofertas de NYK, K-Line y MOL. En segundo lugar, las empresas de Taiwan, poseen alianzas con empresas europeas. Son los casos de Evergreen con sus vínculos con Italia Maritima y con Evergreen Marine, del Reino Unido. O de la propia Yang Ming que opera utilizando sus servicios en España. Las empresas de Corea del Sur establecieron unidades independientes en varios países, abarcando variedad de sectores, hasta su declive y desaparición, como lo mostraron en su día, Hanjin y Hyundai Merchant Marine. Por su parte, las empresas chinas aprovechan su dinamismo económico para reforzar vínculos intra-asiáticos por una parte; y para ampliar su presencia internacional ya sea llevando a cabo adquisiciones de 
empresas (COSCO) ya sea desarrollando estrategias "global carrier" (OOCL). La renovación europea se plasma en las intensas apuestas de expansión de las principales empresas navieras (Maersk, MSC y CMA/CGM) cada vez más presentes en más puertos y sobre todo cada vez más conectadas entre sí.

Esta descripción de las estrategias de las compañías marítimas nos permite subrayar los actuales grados de alianzas entre ellas y entre las compañías europeas. Históricamente, las compañías japonesas y las coreanas se situaban en alianzas diferentes. Sólo la Grand Alliance incluía a compañías europeas, en este caso la alemana Hapag Lloyud. Evergreen era la única empresa que prefería su independencia y no formar parte de ninguna alianza. Finalmente, la compañía china CSCL también estaba fuera de alianzas pero manteniendo acuerdos puntuales con diversas compañías. De este modo, era fácil colegir que la formulación de las alianzas marítimas estaba revelando la apuesta por parte de las compañías asiáticas de un nuevo método para mejorar y racionalizar los servicios y las escalas en la anterior fase de intenso crecimiento y de concentración de tráficos en las líneas Este-Oeste (Frémont \& Soppé, 2004; Zhen et al., 2015; Farrel, 2012).

Se puede decir que, a día de hoy, la actual red de líneas, rutas, puertos y escalas está siendo dominada por una estandarización del método de las compañías asiáticas. Los ejemplos mostrados tanto para COSCO y OOCL, son muy parecidos. Sin embargo, las líneas, rutas, puertos y escalas que utilizan las compañías japonesas agrupadas en ONE (K-Line; NYK y MOL) junto a Evergreen muestran posicionamientos diferentes: incluyen puertos de América Latina y África y mantienen contactos con puertos europeos distintos de los correspondientes al Range Northern; amplían las conexiones por el Mediterráneo; y desarrollan las conexiones con Oceanía. Esto es, apuestan por la búsqueda de un mercado más amplio; tratando de incrementar el poder de negociación y acceder a una red global.

El cambio de actitud de las nuevas compañías se debe a que históricamente las compañías marítimas de líneas regulares estaban consideradas como una herramienta al servicio de la producción orientada a la exportación y estaba siendo considerada como una actividad de outsiders. En la actualidad, la maduración sectorial y la relevancia de la actividades comerciales permite la reorientación de las estrategias, ampliando los cometidos conservando las posiciones concurrenciales adquiridas.

El nuevo mileno ha permitido introducir dos nuevas consideraciones relevantes. La primera fue la constatación de la formalización de estrategias de empresas bajo los principios de cubrir la red global; esto es, de cubrir y abarcar un reparto más homogéneo en lo que respecta a los estándares tradicionales; y, la segunda, es la consolidación de hubs dedicados, especializados y situados en las principales líneas y rutas. (Notteboom et al., 2017). El caso de Maersk 
muestra una tendencia hacia esta última concepción, conexionando todas las rutas, ampliando el número de puertos y ofertando servicios en todo el planeta. Igualmente, dicha apuesta es seguida por la Mediterranean Shipping Co (MSC) y por la francesa CMA-CGM. Estas tres compañías buscan sus estrategias en la consolidación de hubs que permitan imprimir, desde sus puertos principales, un impulso al negocio marítimo y terrestre. Maersk busca la cobertura mundial de los mercados, MSC posee una fuerte presencia en las rutas secundarias; y CMA-CGM se hace fuerte en los mercados complementarios.

\section{LOS DESARROLLOS Y LA RIVALIDAD PORTUARIA}

Establecer algún mecanismo que nos determine la estructura de las redes del transporte marítimo y el papel que desempeñan los puertos en las mencionadas redes es el objetivo de este epígrafe.

Al elaborar las distintas tipologías portuarias, los puertos se pueden clasificar en distintos tipos. El modelo de Taaffe (Taaffe et al., 1963) muestra un proceso de desarrollo que va desde una estructura de puertos pequeños y desconectados a una red formada por uno o dos puertos principales conectados a puertos secundarios que forman un sistema de transporte integrado. Hayuth (1981), entre otros, al desarrollar esta idea introduce los centros de carga (load centres). Notteboom (1997) incorpora el concepto de carga de trabajo portuaria, asociada a varios criterios, tales como puerto de atraque regular para servicios "round the world”; movimientos de grandes volúmenes de contenedores (más de 4.000 TEUS); elevadas cifras de tránsito; y aumentos sustanciales de las cuotas de mercado del puerto en un área determinada. Para Notteboom un centro de carga ha de cumplir por lo menos tres de estos cuatro criterios.

Tanto O’Kelly \& Millar (1994) como Notteboom (2002) han utilizado el término de hubs de transporte, en el sentido que los hubs son aquellos lugares portuarios en los que se realizan numerosos transbordos (mar-mar) mientras que los centros de carga se relacionan con aquellos puertos que poseen un mayor acceso a su área de influencia o hinterland. Baird (2002), por su parte, los utiliza indistintamente.

Heaver et al. (2001) distingue tres tipos de centros de carga. La primera categoría es aquella que abarca a los grandes centros de carga internacionales, grandes dominadores del transporte mundial. Las otras dos categorías de centros de carga incluyen a los puertos medianos (ligados a los intercambios de áreas regionales) y los puertos pequeños (que solo influyen en el comercio nacional). Por tanto, estamos desarrollando una tipología en la que se distinguen los "ejes globales"; los "centros de carga", los "puertos regionales" y los "puertos secundarios"; y sus clasificaciones están en función de la localización de dichos puertos con respecto a las principales rutas marítimas. 
Recientemente, de Langen et al. (2002) utilizan para la identificación de los diferentes tipos portuarios los siguientes elementos: a) lógica de la localización, esto es la posición geográfica de un puerto dentro de la propia red de transporte. Aquí es preciso juzgar y evaluar tanto los componentes de las redes marítimas como aquellas que están relacionadas con las del hinterland; b) los servicios al hinterland; es decir, advertir las cuestiones que desempeñan un papel muy relevante, tales como la importancia de los transbordos (mar-mar), el tamaño del hinterland y las conexiones intermodales del propio hinterland; y c) finalmente las características de los servicios, que vienen justificados por los volúmenes de producción, tamaño y frecuencia de los servicios.

La clasificación nos permite jerarquizar los sistemas marítimos y portuarios de manera muy esquemática. Por ejemplo, los "Ejes Globales" son puertos situados cerca de las principales rutas marítimas e integradas en las redes y ejes de transporte internacional E-W, que conectan las tres áreas más desarrolladas del mundo, América del Norte, Europa y Asia. Mueven los mayores volúmenes y utilizan los buques de mayor tamaño. La principal función de los puertos es servir de tránsito (más del $60 \%$ de las cargas) y potenciar el desarrollo intermodal desplazando las cargas hasta cerca de 300 kilómetros del puerto en la medida que las cargas locales son reducidas. La producción mínima de un eje global es de 600.000 Teus; los buques que sirven a los puertos superan los 5.000 Teus; y las frecuencias de paso son dos veces semanales.

Los "centros de carga" corresponden a los puertos situados en la periferia de las rutas principales E-W de las redes marítimas internacionales, Sirven a un hinterland muy amplio; atraen servicios de primera clase; el transporte de tránsito es menos relevante que en los correspondientes a los ejes globales (no superan el 40\%); y las cargas con destino el hinterland son elevadas (más del $60 \%$ ). Por tanto, dichos puertos se caracterizan por el gran número de conexiones intermodales con el hinterland. En los puertos atracan buques entre 6.000-14.000 Teus; el movimiento mínimo es de un millón de Teus; existen políticas de atracción por parte y hacia las compañías navieras; y predominan las desviaciones de tráficos que son compensadas por los grandes volúmenes transportadas.

Los puertos medianos, por su parte, sirven a aglomeraciones de tamaño considerable, sus hinterlands están más alejados de la costa (hasta 500 kilómetros del puerto). Los buques utilizados oscilan entre 2.000-6.000 TEUS; los volúmenes de carga en los mencionados puertos ascienden a 150.000/200.000 Teus; y la frecuencia es de un servicio por semana.

Analizando las redes internacionales del transporte marítimo y los flujos comerciales se establece una jerarquía portuaria de primer nivel en lo que concierne a los ejes globales y centros de carga y puertos regionales. Destaca la preeminencia de los lugares del sudeste asiático en los ejes globales y centros de 
carga; siendo Europa el continente que presenta una estructura más concentrada en centros de cargas y puertos secundarios (Notteboom, 2002), en tanto que Asia amplia la red de puertos a lo largo de su perímetro costero. En la medida que no todos los puertos de una misma área pueden tener como objetivo convertirse en centros de carga o hubs ya que su número está limitado (Beddow, 2004), el hecho de seleccionar aquellos puertos que están incluidos en las líneas del transporte supone subrayar una jerarquía sobre los restantes en lo que concierne a los flujos comerciales (Bernhofen et al., 2016).

La elección de los puertos de atraque está directamente relacionada con los transportistas y los mercados. La expansión hacia nuevos mercados trae consigo un inevitable aumento de nuevos puertos y al mismo tiempo una racionalización y concentración de tráficos. El reajuste llevado a cabo en lo tocante a los servicios portuarios, coincidiendo con la formación de alianzas marítimas, supuso una nueva búsqueda a la hora de proporcionar servicios a cada área territorial, constituyendo dichos servicios multi-portuarios la característica operacional más relevante de las alianzas marítimas en cada zona geográfica. Esta racionalización e integración de los servicios ha exigido una mayor coordinación de los recursos para proporcionar oportunidades para captar nuevos mercados. Por eso, cada vez son más parecidas las estrategias de las compañías marítimas y, al mismo tiempo, se refuerza la jerarquía de los puertos, con lo que las diferencias entre los primeros puertos del mundo son cada vez más escasas.

Los últimos estudios nos presentan dos importantes dinámicas. La primera corresponde a la expansión y fuerte crecimiento del número de puertos que forman parte de los servicios y de las compañías marítimas. En la década de los noventa, los puertos servidos por las compañías de las alianzas marítimas han aumentado en 470, pasando de los 1.336 en 1989; a los 1.521 en 1994 y alcanzar los 1.806 en 1999 (Slack, Comtois \& McCalla,2002). Dicha distribución revela que casi todas las compañías han incrementado su oferta portuaria y sus servicios marítimos en la mayor parte de las áreas mundiales. Pero lo significativo es el número de puertos que es utilizado semanalmente por las compañías marítimas. Aquí el resultado es espectacular: de 310 puertos en 1989, se pasa a 571 en 1994 y se logra una cifra record en 1999 con 1.249 puertos que poseen un servicio semanal de transporte. Para 2017, los datos conocidos muestran, por ejemplo, que Maersk opera en 76 puertos de 95 países diferentes; MSC tiene relación directa con 35 terminales, en 22 países distintos; CMA-CGM lo hace en 13 terminales alrededor del mundo; los buques de COSCO escalan en 30 puertos mundiales; NYK opera en terminales de 23 puertos. O sea, un fuerte incremento de los servicios, mayor diversificación de la oferta y extensificación de las redes de transporte lo que facilita las conexiones portuarias con los hinterlands más próximos contribuyendo a reforzar el desarrollo económico y las interrelaciones 
económicas de las demandas industriales y de servicios de las respectivas áreas territoriales.

La importancia de las terminales portuarias es cada vez mayor. Actúan anticipándose a las tendencias y a las coyunturas económicas, posicionándose en aquellas ubicaciones de fuerte potencial económico y en las rutas comerciales de mayor intensidad de tráfico, al objeto de constituir lugares de transbordo que acompañan a las políticas de redes y de los corredores multimodales. Las decisiones de la ubicación se lleva a cabo siguiendo tres elementos básicos: a) estar situadas en las redes de las líneas/rutas marítimas y con enlaces a los corredores de los ferrocarriles; b) búsqueda de economías de escala tanto en lo que atañe a las utilización del buque como en lo que respecta a las operaciones de gestión portuaria, incidiendo en las cuestiones de organización, gestión, aspectos técnicos y humanos, etc.; y c) capacidad de constituirse en plataformas logísticas.

En consecuencia, las lógicas del desarrollo y de la implantación se mueven bajo los dos criterios, el del desarrollo propiamente dicho y el de la organización y racionalización. Por el primero, se organizan los flujos de transporte de los clientes bajo la óptica de un operador logístico global, con ventanilla única; y, por el segundo, se acepta la existencia de subcontratación en algunas de las operaciones de la cadena logística. Siguiendo los trabajos de Notteboom \& Winkelmans (2001), Notteboom (2002) y los informes publicados por Ci-online, los procesos de concentración del tráfico contenedorizado y que afectan al entorno portuario son muy variados, tal y como expresamos en la Tabla 7.

Finalmente, se presencia una combinación entre la organización marítimaportuaria con la estructuración espacial definida por las redes ferroviarias. La apuesta a favor de la intermodalidad mar/ferrocarril puede ser identificada atendiendo a dos ejes: al contexto reglamentario y a las decisiones y prácticas de los agentes económicos. En primer lugar, asistimos a una liberalización en lo que concierne a los trayectos del ferrocarril que favorece la libertad de las condiciones de expansión del FFCC, mejorando su productividad y abandonando las líneas no rentables. De otra parte, los agentes económicos están actuando de manera tal que se convierten en empresas multimodales para ganar en eficacia, ofertar todas las facilidades posibles parea poseer ventanillas únicas de gestión y para formalizar los vínculos de complementariedad entre las compañías ferroviarias con otras compañías en la estructuración definitiva de una red.

De esta manera, la liberalización de los servicios ferroviarios forma parte de los ámbitos de competencia que presentan los puertos europeos. Dicha dinámica se desarrolla a distintos niveles y en distintas estrategias. Los puertos del norte de Europa buscan la distribución de las mercancías a escala continental y para ello su radio de influencia sobrepasa el territorio en el que se ubica en puerto, cubriendo, en consecuencia, los mercados del sur y este europeo. Por su parte, los puertos del sur-europeo plantean otra lógica, caracterizada por el 
aprovechamiento de las ventajas del "transit time"; o sea, ganar tiempo en la distribución de las mercancías hacia los centros continentales evitando el poder de atracción de los mega-puertos del Range Northern.

Tabla 7

Factores que afectan al entorno portuario

\begin{tabular}{|c|c|c|}
\hline Dinámicas & Efectos & Resultados \\
\hline $\begin{array}{l}\text { Alianzas marítimas y } \\
\text { redes inter- } \\
\text { empresariales }\end{array}$ & $\begin{array}{l}\text { Las compañías navieras llevan a } \\
\text { cabo procesos de fusión y } \\
\text { adquisición de empresas que } \\
\text { refuerzan su rol y permiten consolidar } \\
\text { líneas marítimas de cara a minimizar } \\
\text { costes y ampliar cuotas de mercado. }\end{array}$ & $\begin{array}{l}\text { Las líneas marítimas se convierten en } \\
\text { proveedores de redes globales. } \\
\text { Una alianza marítima puede mover cargas } \\
\text { libremente en el mercado global. }\end{array}$ \\
\hline Tamaño de los buques & $\begin{array}{l}\text { Los buques de gran tamaño se } \\
\text { construyen para alcanzar economías } \\
\text { de escala. }\end{array}$ & $\begin{array}{l}\text { No todos los puertos pueden servir de } \\
\text { base a los Mega-navíos transoceánicos } \\
\text { debido a los límites de profundidad } \\
\text { requeridos a los recintos portuarios. }\end{array}$ \\
\hline Intermodalidad & $\begin{array}{l}\text { Los hub intermodales terrestres } \\
\text { permiten aumentar el envio de } \\
\text { contenedores a través del continente } \\
\text { para establecer más conexiones con } \\
\text { otros puertos. }\end{array}$ & $\begin{array}{l}\text { Se asiste a nuevas expansiones en lo que } \\
\text { concierne a hinterland y a los forelands de } \\
\text { los puertos. }\end{array}$ \\
\hline $\begin{array}{l}\text { Cambios en la } \\
\text { gobernanza }\end{array}$ & $\begin{array}{l}\text { Las Autoridades Portuarias } \\
\text { experimentan cambios institucionales } \\
\text { notables; pasan del modelo público al } \\
\text { modelo landlord. }\end{array}$ & $\begin{array}{l}\text { Se promueve una toma de decisiones más } \\
\text { ágiles y efectivas por parte de las } \\
\text { Autoridades Portuarias. }\end{array}$ \\
\hline $\begin{array}{l}\text { Competencia entre } \\
\text { puertos }\end{array}$ & $\begin{array}{l}\text { Los puertos con mayores niveles de } \\
\text { proximidad geográfica aumentan su } \\
\text { independencia usando la } \\
\text { combinación de estrategias } \\
\text { competitivas. }\end{array}$ & $\begin{array}{l}\text { La competencia desequilibrada llevada a } \\
\text { cabo por los puertos vecinos es moderada, } \\
\text { mientras que sus estrategias contrastan en } \\
\text { el marco de una competencia } \\
\text { internacional. }\end{array}$ \\
\hline $\begin{array}{l}\text { Sostenibilidad portuaria } \\
\text { y desempeño ambiental }\end{array}$ & $\begin{array}{l}\text { Los puertos tienen cada vez más } \\
\text { desafíos en lo que respecta a la } \\
\text { eficiencia y a sus estándares } \\
\text { relativos a la sostenibilidad y defensa } \\
\text { de los parámetros medio- } \\
\text { ambientales. }\end{array}$ & $\begin{array}{l}\text { Las Autoridades Portuarias comienzan a } \\
\text { concebir estrategas verdes y a planificar } \\
\text { acciones para estar en armonía con el } \\
\text { entorno local y con todos los miembros de } \\
\text { la cadena de suministro. }\end{array}$ \\
\hline
\end{tabular}

Fuente: Elaboración propia.

La nueva competencia marítima y ferroviaria incita a la complementariedad de dichas organizaciones. Esta nueva composición de las redes de distribución y conexión convierte al transporte de mercancías por vía férrea como otro de las objetivos de la política marítima, en la medida que privilegia tanto lugares geográficos como estimula a los agentes económicos en su interés de ampliar sus radios de influencia, valorizando los distintos de distribución y organización terrestre.

En consecuencia, presenciamos una doble lógica. Los puertos estructuran líneas/rutas, en los que se concentran los tráficos de mercancías favoreciendo la polarización en determinados centros; en tanto que los servicios ferroviarios practican una estructuración axial que se opone a la lógica de la integración y de redes, al conectar solamente un punto a otro. Por tanto, la puesta en práctica 
llevada a cabo, hasta el momento, determina que los operadores buscan y potencian "redes de concentración" que supongan una relación directa entre puerto y territorio; esto es, situarse entre la eficacia de los servicios ferroviarios y las líneas estratégicas de los puertos.

\section{CONCLUSIONES}

Apreciamos una creciente y más intensa competencia portuaria que se manifiesta por los conceptos derivados de las ventajas comparativas y por las diferentes concepciones derivadas de los "niveles de gobernanza" de las autoridades portuarias (Chen \& Everett, 2014). Sus objetivos radican en maximizar los tráficos y sus rendimientos.

Presenciamos, en primer término, un aumento significativo de la demanda de instalaciones y de servicios portuarios y los puertos entran en el nuevo mercado de oferta. En segundo lugar, asistimos a una intensa y creciente movilidad de los operadores que requieren de instalaciones portuarias específicas y de terminales fijas en los puertos, reforzando los criterios de selectividad portuaria en base a la localización y a las bases de sus operaciones. En tercer lugar, las nuevas condiciones de la competencia obedecen a las novedosas estrategias de los operadores subrayando una nueva jerarquía portuaria. Asistimos a cambios sustanciales en lo que concierne al tamaño de los buques y al tráfico marítimo; y a la organización de la industria del transporte marítimo, por medio de la potenciación de la integración vertical, el control de los ciclos logísticos e intermodales y el outsourcing logístico. El mercado portuario asiste a una mayor competencia entre puertos, precisando políticas encaminadas a reducir las tarifas, logro de menores tiempos portuarios, y reducción de los riesgos derivados de la sobrecapacidad. Asimismo, presenciamos un desarrollo de las nuevas tareas y funciones ligadas a la redistribución de industrias orientadas al puerto y unas conexiones más estrechas del puerto con otros medios de transporte. Por tanto, la competencia portuaria lleva a una selección portuaria, define los nuevos actores, diseña las nuevas ventajas competitivas y subraya las condiciones que han de presentar los operadores portuarios bajo los nuevos elementos que definen las ventajas competitivas.

Las principales terminales portuarias presentan un notable proceso de cambio y de adaptación que está dirigido a los siguientes objetivos: a) aumento de la responsabilidad de la terminal sobre los requisitos del buque; b) control de los factores exógenos que influyen en los tiempos de tránsito en los puertos; c) admisión de buques de mayor tamaño; d) logro de mayores economías de escala y de mayores densidades para el transportista; y e) promueve ciclos hub-andspoke, en lugar de conexiones directas por medio de la colaboración entre compañías estibadoras y compañías navieras. 
Este nuevo desarrollo portuario supone incidir sobre el tiempo del tránsito (mejor planificación del servicio y reducción de las incertidumbres sobre las llegadas de los buques) y sobre la localidad/fiabilidad de los servicios (servicios menos variables y estandarización de las operaciones de carga y gestión en tierra), lo que suponen una nueva herramienta competitiva para atraer y conservar a los usuarios del puerto y una racionalización de las cadenas de distribución.

A medio plazo las perspectivas del transporte marítimo dependerán de la evolución de la economía mundial. La nueva organización y expansión empresarial nos permite observar una concentración del armamento portuario (China, Corea y Japón representan en la actualidad más del 50\% del armamento de contenedores) y la constitución de Mega-empresas que aceleran la mencionada concentración, inclinándose hacia un tráfico Este-Oeste y abriendo nuevas escalas (Boyes, 2004). Esta concentración de escalas portuarias incide de manera directa en la organización portuaria.

Dicha nueva especialización convierte a los puertos en verdaderos centros de distribución, extendiendo su respectiva zona de influencia terrestre (Hinterland) y marítima (foreland); y acentuando la rivalidad entre instalaciones. En consecuencia, el horizonte de los puertos se centra en conseguir altas tasas de productividad; y para ello deben: a) especializar las terminales portuarias para responder con eficiencia a la demanda de nuevos servicios; b) desarrollar una red de puertos secos para posibilitar la extensión de zonas influencia de cada puerto, lo que exige poseer una buena conexión intermodal que reduzca los costes; c) afrontar la construcción de zonas de actividades logísticas, para aprovechar el intermodalismo y para aumentar el valor añadido de las mercancías; d) poseer planes de calidad que garanticen la fidelización de los clientes; y e) desarrollar sistemas de información electrónica. En consecuencia, los puertos deben dar respuesta a los servicios globales y formar parte de los corredores de transporte.

\section{REFERENCIAS BIBLIOGRÁFICAS}

BAIRD, A.J. (2002). "Privatization trends at the world top-100 container ports". Maritime Policy and Management. 29(3), 271-284.

BEDDOW.M. (2004). "Hub Port Ambitions". Containerisation International, $\mathrm{n}^{\circ}$ may. 79-83.

BERNHOFEN D.M., EL-SAHLI, Z., KNELLER, R.(2016). "Estimating the effects of the container revolution on world trade". Journal International Economics. 98, 36-50

BOYES, J.R.N. (2004). "Chinese Crackers". Containerisation International, nº march, 8586.

BROOKS, M.R. (2004)."The governance structure of ports". Review of Networks Economics. 3 (3), 168-183. 
BROOKS, M.R., CULLINAME, K, PALLIS, A. (2017). "Revisiting port governance and port reform a multi-country examination". Research Transportation Business \& Management. 22. 1-10.

CHEN, S-L., EVERETT, S. (2014). "The dynamics of Port Reform. Different contexts, Similar Strategies". Maritime Policy and Management. 41(3). 228-01.

DUCRUET, C., ZAIDI, F. (2012). "Maritime Constellations: a complex network approach to shipping and Ports". Maritime Policy and Management. 39(2). 151-168.

DUCRUET, C. (2016). "The polarization of global container flows by inter-oceanic canals: geography coverage and network vulnerability". Maritime Policy and Management. 43(2). 242-260.

EL KALLA, M., ZEC, D., JUGOVIC, A. (2917). "Container ports competition in light of contemporary liner shipping market dynamics". Scientific Journal of Maritime Research, 31. 128-136.

FARREL, S. (2012). "The ownership and management structure of container terminal concessions". Maritime Policy and Management, 39 (1), 7-26.

FRÉMONT, A., SOPPÉ, A. (2004). Les stratégies des armateurs de lignes régulières: la desserte maritime entre domination asiatique et renouveau européen. Seminaire Maritime INRETS, 9, janvier.

GONZALEZ-LAXE F., FREIRE, Ma. J.; PAIS, C. (2012). "Maritime degree, centrality and vulnerability: Port hierarchies and emerging areas in containerized transport". Journal of Transport Geography. 24, 33-44.

GUERRERO, D., RODRIGUE, J.P. (2014). "The waves of containerisation shifts in global maritime transportation". Journal of Transport Geography. 34. 151-164.

HARALAMBIDES, H.E. (2017). "Globalisation. Public sector reform and the role of Ports in international supply chains. Maritime Economics \& Logistics. 19(1). 1-51.

HAYUT (1981). "Containerisation and the load centre concept". Economic Geography. 57. 160-176.

HEAVERT,T., MEERSMAN;H., VAN DE VOORDE, E. (2001). "Co-operation and competition in international container transport: strategies for ports". Maritime Policy and Management. 28(3). 293-305.

HEAVER, T. D. (2002). "Supplied chain and logistics management: implications for liner shipping", in C. Grammenos (Ed). The Handbook of Maritime and Economic Business. London .LLP. 375-396.

HESSE, M., RODRIGUEZ, J. P. (2004)."The Transport geography of logistics and freight distribution". Journal of Transport Geography. 12. 71-184.

HIRATA, E. (2017). "Contestability of container liner shipping market in alliance Era". The Asian Journal of Shipping and Logistics. 33(1) 27-32.

HOYLE,B.S. \& HILLING,D (ed.) 1984. Seaport Systems and Spatial Change, Technology, Industry and Development Strategies. Chichester. John Wiley \& Sons.

LANGEN, P. W.de; et al. (2002). A stylised container port hierarchy: a theorical and empirical exploration. IAME Panamá 2002 Conference Proceedings.

MERK, O., BUSQUET, B., ARONIETI, R. (2015). The impact of megaships. International Transport Forum. OCDE. Paris.

NG, A. (2018). "Geography and institutional change: insights from a container terminal operator". Maritime Economics \& Logistics. (in press). 
NOTTEBOOM, Th (1997). "Concentration and the load center development in the European container port system". Journal of Transport Geography. .5. 99-115.

NOTTEBOOM, Th. (2002). "Consolidation and contestability in the European container handling industry". Maritime Policy and Management. 29(3). 257-269.

NOTTEBOOM, Th., WINKELMANS, W. (2001). "Structural changes in logistics: how will port authorities face the challenge?". Maritime Policy and Management. 28(1). 71-89.

NOTTEBOOM, Th. RODRIGUE, J.P. (2012). "The corporate geography of global container terminal operator". Maritime Policy \& Management. 39(3). 249-279.

NOTTEBOM, Th., PAROLA, F., SATTA, G., PALLIS, A. (2017). "The relationship between port choice and terminal involvement of alliance members in container shipping". Journal of Transport Geography, 64, 158-173.

O' KELLY, M.E., MILLER, H.J. (1994)."The Hub Network design problems: a review and synthesis". Journal of Transport Geography. 2. 31-40.

PAROLA, F., SATTA, G., PANAYIDES, P.M. (2015). "Corporate strategies and profitability of maritime logistics firms". Maritime Economics \& Logistics, 17 (1). 52-78.

SLACK,B., COMTOIS, Cl.; \& MCCALLA, R. (2002). "Strategic alliances in the container shipping industry: a global perspective". Maritime Policy and Management. 29 (1). 65-76.

SYS, C. MEERSMAN, H., VAN DE VOORDE, E. (2011). "A non-structural test for competition in the container liner shipping industry". Maritime Policy \& Management, 38(3). 219-234

TAAFFE, E.J.; MORRIL, R.L., GOULD, P. R. (1963). "Transport Expansion in Underdeveloped Countries: a comparative analysis". Geography Review. 53. 503-529.

TOURRET, P (2004). Les armements asiatiques de la conteneurisation. ISEMAR, Note de Synthése. $\mathrm{n}^{\circ} 68$. octobre.

UNCTAD (1992). Ad Hoc Intergovernmental Group of Port Experts. Port Marketing and the Third Generation Port. Geneve.

YEO, H.J. (2013). "Geography of mergers and adquisitions in the container Shipping industry". The Asian Journal of Shipping and Logistics. 29(3). 291-314.

YEO, H.J. (2015). "Participation of private investors in container terminal operation: influence of global terminal operators". The Asian Journal of Shipping and Logistics. 31(3). 363-383.

ZHENG,, J., GAO, Z., YANG, D., SUN, Z. (2015). "Network design and capacity exchange for liner alliances with fixed and variable container demands". Transportation Science. 49(4). 886-899. 


\section{Anexo}

Clasificación de los diez primeros puertos de contenedores del mundo (expresados en miles de Teus)

\begin{tabular}{|c|c|c|c|c|c|c|c|c|c|}
\hline & \multicolumn{3}{|c|}{1980} & \multicolumn{3}{|c|}{1990} & \multicolumn{3}{|c|}{2000} \\
\hline & Puerto & Pais & Teus & Puerto & Pais & Teus & Puerto & Pais & Teus \\
\hline 1 & N.York/NJ. & EEUU & 1947 & Singapur & Singapur & 5223 & HongKong & China & 18098 \\
\hline 2 & Rotterdam & P. Bajos & 1900 & HongKong & China & 5100 & Singapur & Singapur & 17087 \\
\hline 3 & HongKong & China & 1464 & Rotterdam & P. Bajos & 3666 & Kaohsiung & Taiwan & 7426 \\
\hline 4 & Kaohsiung & Taiwan & 979 & Kaohsiung & Taiwan & 3494 & Busan & CoreaSur & 6383 \\
\hline 5 & Singapur & Singapur & 916 & Kobe & Japón & 2595 & Rotterdam & P. Bajos & 6290 \\
\hline 6 & Hamburgo & Alemania & 783 & L.Ángeles & EEUU & 2587 & Shanghai & China & 5612 \\
\hline 7 & Oakland & EEUU & 782 & Busan & CoreaSur & 2348 & L.Ángeles & EEUU & 4879 \\
\hline 8 & Seattle & EEUU & 781 & Hamburgo & Alemania & 1968 & L.Beach & EEUU & 4601 \\
\hline 9 & Kobe & Japón & 727 & N.York/NJ. & EEUU & 1871 & Hamburgo & Alemania & 4248 \\
\hline \multirow[t]{3}{*}{10} & Amberes & Bélgica & 724 & Keelung & Taiwan & 1828 & Amberes & Bélgica & 4082 \\
\hline & \multicolumn{3}{|c|}{2006} & \multicolumn{3}{|c|}{2012} & \multicolumn{3}{|c|}{2017} \\
\hline & Puerto & Pais & Teus & Puerto & Pais & Teus & Puerto & Pais & Teus \\
\hline 1 & Singapur & Singapur & 24792 & Shanghai & china & 32529 & Shanghai & China & 37133 \\
\hline 2 & HongKong & China & 23320 & Singapur & Singapur & 31649 & Singapur & Singapur & 30903 \\
\hline 3 & Shanghai & China & 21710 & HongKong & China & 23117 & Shenzhen & China & 23979 \\
\hline 4 & Shenzhen & China & 18468 & Shenzhen & China & 22940 & Ningbo & China & 21560 \\
\hline 5 & Busan & CoreaSur & 12030 & Busan & CoreaSur & 17046 & HongKong & China & 19813 \\
\hline 6 & Kaohsiung & Taiwan & 9774 & Ningbo & China & 15670 & Busan & CoreaSur & 19800 \\
\hline 7 & Rotterdam & P.Bajos & 9690 & Guangzhou & China & 14743 & Guangzhou & China & 18857 \\
\hline 8 & Dubai & UEA & 8923 & Qingdao & China & 14503 & Qingdao & China & 18010 \\
\hline 9 & Hamburgo & Alemania & 8861 & Dubai & UEA & 13270 & Dubai & UEA & 14772 \\
\hline 10 & L.Ángeles & EEUU & 8469 & Tianjin & China & 12300 & Tianjin & China & 11490 \\
\hline
\end{tabular}

Fuente: Elaboración propia, siguiendo los datos proporcionados por Lloyd’s List. 